Durch Einleiten von trocknem Salzsăuregas in die methylalkobolische Löвung der Sänre erhält man den Nitroveratrumsăuremethylester rom Schmp. 143-144".

Die von uns aus Nitroranillinmethyläther dargestellte Nitroveratrumsäure erwies sich somit identisch mit jener, welche Tiemann. und Matsmoto durch Nitriren von Veratrumsăure erbalten haben.

504. W. Muthmann und L. Stütz el: Fine einfache Mothode. zur Daratellung der Schwefel-, Chlor- und Brom-Verbindungen. der Ceritmetalle.

(Eingegangen am 30. November.)

1. Sulfide.

Die Literatur äber die Schwefelverbindungen der seltenen Erden. ist eine recht dürftige. $Z$ war liegt eine Anzahl von Angaben verschiedener Forscher über diese Körper vor, -doch dürfte keiner derselben sie in einigermaassen reinem Zustande bis jetzt dargestellt haben. Die alten Versuche ron Mosander and Berzelius') bedürfen schon deshalb einer Revision, weil danıals die Trennungsmethoden noch nicbt gut ausgearbeitet waren und die Genannten bei. ibren Versuchen wohl zweifellos von Erdgemischen ausgingen; ein Gleiches gilt von den Angaben von Frerichs und Smith?, sowie von $\operatorname{Lange}{ }^{3}$ ).

Die sorgfaltigsten Versuche über unsere Körper sind wohl von Didier") ausgefährt worden. Derselbe erhitzte Cerdioxyd resp. Lantbanoxyd im Schwefelwasserstoffstrom auf ca. 600" und erhielt anf diese Weise Producte, welche nach seiner Angabe sauerstofffrei waren. Doch geht die Umsetzung, wie wir uns überzeugt haben,. nur sehr langeam vor sich; besonders das Cerdioxyd verwandelt sich nur dann einigermassen vollstăndig in Cersulfid, wenn es als änsserstfeines Pulver rerwendet wird. Ein ganz sauerstofffreies Práparat konnte der Eine von uns, der schon vor 2 Jahren sich mit der Fragebeschäftigte, nach Didier's Methode überbaupt nicht erbalten.

Wir haben nun eine ansserst bequeme und einfache Methode zurHerstellung der Sulfide benutzt, welche auf Umsetzung der wasserfreien Sulfate im Schwefelwasserstoffstrom beruht. Da man, wie später năher gezeigt werden soll, die Sulfide sehr leicht und quantitativ in wasserfreie Chloride und Bromide überführen kana, so ist damit

1) Berzelius, Lehrb., 5. Aut. 2, $: 93$.

3) Joun. f. prakt. Chem. 8ะ, 132.

3) Ann. d. Chem. 19', 359.. †) Compt, rend. 100, 1462. 
auch eine bequeme Darstellungswethode der Letzteren ans den ja sebr leicht zo orbaltenden Sulfiten gegeben.

Als Ausgangematerial dienten folgende Salfate:

$$
\begin{gathered}
\mathrm{Ce}\left(\mathrm{SO}_{4}\right)_{2} .8 \mathrm{H}_{2} \mathrm{O}, \underset{\text { uad }}{\mathrm{La}}\left(\mathrm{SO}_{4}\right)_{3}, 9 \mathrm{H}_{8} \mathrm{O}, \mathrm{Nd}_{2}\left(\mathrm{SO}_{4}\right)_{3} .8 \mathrm{H}_{2} \mathrm{O} \\
\text { ard } \mathrm{Sr}_{4} \cdot 5 \mathrm{H}_{2} \mathrm{O} \text {. }
\end{gathered}
$$

Die Salze wurden durch Erbitzen wusserfrei gemacht, im Porzellanschiffchen in einer Verbrennungsröhre bis zar Gewichtoconstanz scbwach geglüht und dann bei begimender Rothgluth im trocknen Schwefelwasserstoffstrom längere Zeit erhitzt. Sehr bald macht sich eine Aenderung der Farbe bemerkbar, und es resultirt beim Cer ein braunscbwarzes bis ochwarzes, beim Lanthan ein rein gelbes, beim Neodym ein olivengränes und beim Praseodym ein bellcbokoladenfarbenes Pulver. Bei der Herstellung grōsserer Mengen der Sulfide, z. B. zur Gewinuang von $8 \theta \mathrm{g}$ wasserfreien Cerchloride, wurde das wasserfreie Sulfat ohne Weiteres in eine nöglichst weite Verbrennungsröbre gegeben and erhitzt. Die Ueberfährung ist so gut wie quantitativ; die kleinen Differenzen bei nachfolgenden Zahlen erklären sich darans, dase die wasserfreien Sulfate wăhrend des Wagens Wasser aus der Luft anfnahmen.

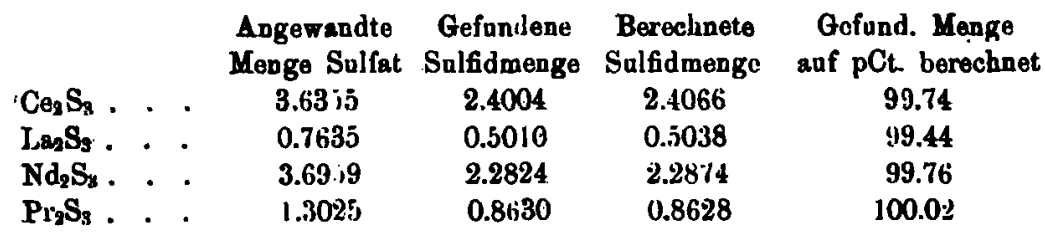

Mit Eiswasser Jängere Zeit geschüttelt und filtrirt, gaben die Sulfide im Filtrat mit Chlorbaryum keine Trübung, in Salzažare lösten sie sich beim Kochen fast völlig klar auf.

Die Analysen ergaben folgende Zahlen:
1. Cegs. Ber. S 25.53, Ce 74.47.
Gef. " 25.12, " 74.52.
2. $\mathrm{La}_{2} \mathrm{~S}_{3}$. Ber. S 25.78, La 74.22.
Gef. - 25.63, - 7459 .
3. $\mathrm{Nd}_{3} \mathrm{~S}$ :. Ber. S 25.07, Nd 74.97.
Gef. " 25.26, - 74.82.
4. $\operatorname{Pr}_{2} \mathrm{~S}_{\mathrm{x} .}$ Ber. $\mathrm{S} 25.47, \operatorname{Pr} 7452$.
Gef. $25.39,-74.70$.

Die Ausfäbrong der Analysen geschuh in folgender Weise:

Zur Metallbestimnang wurden tie Sulfide im gewogenen Platintiogel über dem Geblasse $1^{1 / 2}-2$ Standen heftig gaglüht und als 0 xyd gewogen.

Zur Schwefelbestimmugg wurden zwei Methoden benutzt:

1. Nach Doutecom warde 1 Theil Sulfid mit 8 Theilen eines $G_{\theta}$ mischea gleicher Theile von Kaliumchlorat, Natriumcarbonat und Natrinmchlorid erst langeam in einem grossen bedeckten Porzellantiegel bis zum 
Schmelzen erbitzt und dann längere Zeit mit dem Geblüse gegläht: nach dem Erkalten wurde die Schmelze wiederholt mit kochendem Wasser ausgezogen, auf ein Filter gebracht, der Rückstand mehrere Male mit heissem Wasser ausgewaschen und hierauf im angesăuerten Filtrate mit Barjumchlorid gefält.

2. I Theil Sulfid wurde mit 10 Theilen eines Gemenges aus 2 Theilen wasserfreiem, kohlensaurem Natrium und 1 Theil Salpeter vernischt, im Platintiegel allmāhlich zum Schmelzen erhityt nnd hitrauf einige 7eit geglüht; nach dem Erkalten wurde die Schmelze mit Wasser erwärmt und filtrirt; der Rückstand wurde mit reinem kohlensaurem Natrium ausgekocht, der unlösliche Theil mehrere Male ausgewaschen, das Filtrat mit Salzsūure versetzt und zur Vertreibung der Salpetersăure eingedampft, hierauf in Waseer aufgenommen und mit Baryumchlorid gefallt.

Beide Modification'n lieferten gleich gute Resu'tute.

An der Luft halten sich die Sulfide bei gew ïhnlicher Temperatur ziemlich got, und zwar ist die Haltbarkeit un so grösser, je stärker bei der Darstellung erhitzt worden war. Von kochendem Wasser werden sie langsam unter Schwefelwasserstoffentwickelung zersetzt, am leichtesten die Lanthanverbindung. Verdünnte Säuren lösen sehr leicht unter Scbwetelwasserstoffentbindung zu den betreffenden Salzen.

Die Entzündungstemperator an trockner Lnft ist eine relativ niedrige; sie liegt bei allen vièr Snlfiden unterhalb Rothgluth. Cersulfid ist sogar pyrophorisch, wenn es in genügend feiner Vertheilung vorliegt; es ist uns einige Male rorgekommen, dass beim Oeffnen des Verbrennungsrohres das schwarzbraune Product ohne Weiteres Feuer fing und unter Entwickelung von schwefliger Säure verglimmte. Andere Prăparate wieder entzündeten sich nicht von selbst und kounten an der Luft aufbewabrt werden. Aehnliches hat übrigens schon Lange an seinen Gemischen von Cersulfid und Ceroxyd beobachtet.

Das Product der Verbrennung ist bei allen Präparaten ein Gemenge von Oxyd und wasserfreiem Sulfat, welch' letzteres durch Eiswasser extrahirt werden kann. Daraus folgt, dass man bei der quantitatiren Oxydbestimmung stark und andauernd an der Luft glüben muss, um alle Schwefelsäure auszutreiben.

Nach Mosander soll das Cersulfid beim Kochen mit Aetzkali ein grünliches Oxysulfuret geben; wir können diese Angabe nicht bestätigen, da wir auf diesem Wege immer nur gelbliches Cerihydrooxyd erhielten. Ebenso wenig haben wir das krystallisirte Cersulfid Mosander's herstellen kōnnen, welches goldgelbe Schuppen bilden soll; beim Zusammenschmelzun von Cersulfid oder von Cerdioxyd mit Schwefelkalium entstand immer das dunkelbraune, scheinbar amorphe Sulfid.

Ob Mosander unter anderen Bedingungen arbeitete, als wir, oder ob sein Ausgangsmaterial fremde Beimengungen enthielt, möchten wir nicht sicher entscheidev; wabrscheinlich war wobl Letzteres der Fall.

Baricbte d. D. chem. Gesellacbaft. Jabrg. XXXII. 
Die specifischen Gewichte der Sulfide wurden mit dem Pyknometer bestimmt. Die Versuche selbst wurden mit der nöthigen Vorsicht?ausgefübrt, doch standen uns nur kleine Mengen Substanz zur Verfügung, sodass infolge der dadurch bedingten unverneidlicben Fehler die Zahlen als nicht absolut genau zu betrachten sind. Die Versuche wurden bei $11^{\circ}$ uusgeführt. Die specifische Gewichtsbestimmung des Tetrachlorkohlenstoffes, welcher als Flüssigkeit benutzt wurde, ergab bei obiger Temperatur die $Z_{n}$ hl 1.60967 .

Es ergaben sicb folgende Werthe der specifischen Gewichte:

Spec. Gewicht Angew. Menge Atomgewicht

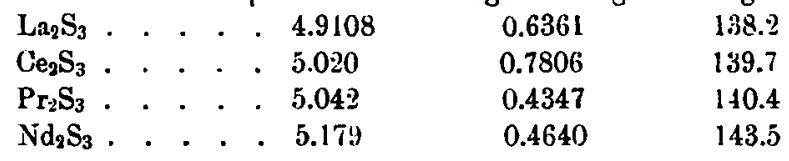

Es lag bis jetzt nur eine specifische Gewichtsbestimmung des Cersulfides von Didier vor, welcher ca. 5.1 angiebt.

Wie man sieht, nehmen die specifischen Gewichte mit der $\mathrm{Zu}$ nahme der Molekulargewichte etwas zu, im Uebrigen sind sie einander sehr ähnlich, wie dies bei Elementen von solchen übereinstimmenden Eigenschaften zn erwarten war.

Von einer Berechuung der Moleknlarvolumina wurde abgesehen, da die Zuhlen doch nicht genau genug waren.

\section{Die Chloride.}

Die oben beschriebenen, leicht zu erhaltenden Schwefelrerbindungen setzen sich mit Salzsäuregas glatt unter Schwefelwasserstoffentwickelung zu den wasserfreien Chloriden um; es ist damit eine Methode gegeben, diese wichtigen Körper schnell in reinem Zustande zu gewinnen, besonders, wenn es sich nicht um zu grosse Quantitäten handelt. Wir verwundelten zu diesem $Z_{w e c k e}$ die Sulfate, wie oben beschrieben, in Sulfide, verdrängten dann in dem etwas abgekühlten Verbrennungsrohr den Schwefelwasserstoff zunächst durch Kohlezdioxyd und dieses durch reinen, scharf getrockneten Chlorwasserstoff, welcher durch Eintröpfeln concentrirter Schwefelsăure in reine concentrirte Salzsäure erbalten worden war. Dann wurden die Flammen des Verbrennungsofens wieder böher gedreht und die Substanzen einige Zeit auf einer Temperatur erhalten, welche beginnende Rothgluth nicht überstieg.

Die Einwirkung beginnt sehr bald, was man an der Farbenänderung der meist dunklen Sulfide erkannt, und es hinterbleibt beim Cer und Lanthan zuletzt ein rein weisses, beim Praseodym ein grūnes und beim Nendym ein prächtig rosa gefärbtes Product. Um die Luftfeuchtigkeit völlig auszuschliessen, luringt man am Ende des Verbrennungsrohres, besonders beim Erkalten, eine Cblorcalciumröhre an. Kleinere Mengen, die gewogen werden sollten, wurden im 
Porzellanschiffchen, grössere direct in das Verbrennungsrohr eingeführt. Einmal haben wir nach dieser Methode $100 \mathrm{~g}$ wasserfreies Cerosulfat in das Chlorid verwandelt; die Operation war in 4 Stunden beendet. Bei Anwendung solch' grosser Quantitäten muss man ein ziemlich langes Verbrenuungsrohr verwenden, da durch Zersetzung des Schwefelwasserstoffs Schwefel sich bildet, der zuletzt, eventuell unter Anwendung eines Kohlendioxydstromes, in das aus dem Ofen berausragende Ende des Rohres hinein zu destilliren ist. Vorwärmen des einzuführenden Gases beschlennigt die Operation ausserordentlich.

Nacbstehende Tabelle giebt die Mengen des angewandten Sulfides und des erbaltenen Chlorides an:

\begin{tabular}{lcccc}
\multicolumn{2}{c}{$\begin{array}{c}\text { Angewandte Menge } \\
\text { von Sulfid }\end{array}$} & $\begin{array}{c}\text { Erhaltene } \\
\text { Chloridmenge }\end{array}$ & $\begin{array}{c}\text { Berechnete } \\
\text { Menge Chlorid }\end{array}$ & Auf pCt. berechnet \\
$\mathrm{Ce}_{2} \mathrm{~S}_{3}$ & 0.2575 & 0.3655 & 0.3638 & 100.46 \\
$\mathrm{~L}_{2} \mathrm{~S}_{3}$ & 0.1307 & 0.1718 & 0.1716 & 100.12 \\
$\mathrm{Nd}_{2} \mathrm{~S}_{3}$ & 0.2273 & 0.2971 & 0.2967 & 100.13 \\
$\mathrm{Pr}_{2} \mathrm{~S}_{3}$ & 0.1286 & 0.1690 & 0.1686 & 100.23
\end{tabular}

Die nur sehr wenig zu hoben Werthe finden ihre Erklärung dadurch, dass die wasserfreien Chloride äusserst begierig Wasser aus der Luft aufnehmen.

Cer- und Lanthan-Chlorid sind, wie oben erwähnt, rein weisse, krystallinische Massen, Neodymchlorid ist rosa gefärbt, Praseodymcblorid grün. Sie nehmen beim Liegen an der Luft sehr scbnell Wasser auf und lösen sich, wenn obige Temperatur bei der Darstellung nicht allzusehr überschritten wird, völlig klar und zwar unter Zischen im Wasser auf; ebenso sind alle in Alkohol löslich. Sie sind sebr schwer flüchtig und verhältnissmăssig leicht schmelzbar.

Die Analyse ergab für das Cerchlorid wach folgenden Zahlen eine Zusammensetzung der Formel $\mathrm{CeCl}_{3}$.

$$
\begin{aligned}
& \mathrm{CeCl}_{3} \text {. Ber. } \mathrm{Cl} 43.20 \text {, Ce } 56.43 . \\
& \text { Gef. " 42.91, " } 56.29 .
\end{aligned}
$$

Zur Analyse wurde das abgewngene wasserfreie Cerchlorid in Wasser gelöst, das Chlor mit Silbernitrat abgeschieden und als Chlorsilber gewogen; in Filtrat wurde das überschūssige Silber mit Salzsäure gefällt und hierauf das Cer mit Ammoniak abgeschieden, geglübt. und als Dioxyd gewogen.

Wir haben selbstredend auch versucht, ob sich nicht durch Einwirkung von Chlorwasserstoff auf die wasserfreien Sulfate direct die Chloride erhalten lassen, konnten indessen sauerstoffifeie Körper so nicht darstellen. Die Sulfate zerfallen bei böherer Temperatur zu leicht in Oxyde und Schwefeltrioxyd; es waren allerdings in einem durch Einwirkng von Chlorwasserstoff auf Sulfat bei ca. $500^{\circ}$ erhaltenen Reactionsproduct nicht unbedeutende Mengen von Chlorid nachweisbar, doch löste sich die Masse nicht klar in Wasser anf. 
Unmöglich ist es nicht, dass bei einer etwas niedriger liegenden Temperatur die Umsetzung glatt erfolgt, doch ist der Umweg über das Sulfid der grösseren Sicherheit wegen vorzuziehen.

Zum Schlusse möchten wir noch kurz die Literatur über den oben behandelten Gegenstand besprechen. Eine der unseren ähnliche Methode scheint schon Mosander ${ }^{1}$ ) zur Herstellung von wasserfreiem Cerchlorid verwendet za haben, doch mit dem Unterschiede, dass er das Sulfid im Chlorstrom erhitzte. Ein von uns angestellter Versuch zeigte, dass die Reaction sehr heftig, unter Feuererscheinung und Zerstäubea der Substanz, ror sich geht; auch springen dabei leicht die Röhren.

Das gewöhnliche Verfahren besteht darin, dass man eine Lōsung der Oxyde in Salzsäure mit Chlorammoninm eindampft und die trockne Salzmasse dann bis zum Verjagen des Chlorammoniums glüht. Man darf bei Anwendung desselben mit dem Salmiak nicht sparen und muss das Trocknen des Salagemisches bei möglichst niedriger Temperatur - auf dem Ilampfbade - ausführen, weil sonst durch die Reaction der Chloride mit Wasserdampf Oxyde sich bilden, genau wie dieses beim Chlormagnesium der Fall ist. Diese Methode dürfte, wenn es sich um Herstellung grösserer Mengen handelt und ein kleiner Sauerstoffgehalt nicht schadet, die bequemste sein. Insbesondere die Kaliumdoppelchloride der Metalle, die zur elektrolytischen Gewinnung der Metalle sich eignen, haben wir immer in dieser Weise hergestellt. Wenn Kilogramme der Chloride gemacht werden sollen, so bedarf man bei Anwendung der Sulfidmethode besonderer Einrichtungen, welche uns nicht zur Verfügung standen.

Von sonstigen Methoden sind noch zu erwähnen die ron L. Meyer ${ }^{2}$ ), Erhitzen von Cerdioxyd im Verbrennungsrohr im Tetrachlorkohlenstoffdampf, und Didier ${ }^{3}$ ), Erhitzen von Dioxyd in einem Gemenge von Kohlenoxyd und Chlor; in beiden Fällen verläuft die Reaction ziemlich träge. Pettersson glühte ein Gemenge der Oxyde mit Kohle im Porzellanrohr im Chlorstrom, wobei die Chloride, wenigstens theilweise, sublimiren; doch müssen wegen der Scbwerflüchtigkeit sehr hohe Temperaturen verwendet werden. Durch Erhitzen der trocknen Oxalate im Salzsäurestrom konnten wir bei zwei Versuchen nur sauerstoffhaltige Körper erbalten, die sich nicht klar in Wasser lösten. Nach unseren Erfahrungen ist die oben beschriebene Methode zur Herstellung reiner Producte die bequemste und sicherste.

\section{Cerbromid.}

Auch zur Darstellung der Bronide kann die beschriebene Methode mit Vortheil benutzt werden, wie aus folgendem Versuch hervorgeht:

1) Berzelius, Lehrbuch, 5. Auf. II, 790.

2) Diese Berichte 20,681. 3) Compt. rend. 101, 882. 
Etwa 3 g Cersultid wurden im Porzellanschiffchen im Verbrennangerohr auf $400-600^{\circ}$ im Bromwasserstoffstrom erhitzt; letzterer wurde nach der üblicben Methode aus Phosphor, Wasser und Brom orhalten. Schou nach 10 Minuten begann das Pulver, sich zu entfärben, und nach 3/4-8töndiger Einwirkung war die Reaction vollendet; es wurde ein schneeweisses, krystallinisches, in Wasser klar lösliches Pulver erhalten, das aus der Luft begierig Feuchtigkeit anzog.

Die Analyse stimmte auf $\mathrm{CeBr}_{3}$ :

Ber. Br 63.16, Ce 36.84.

Gef. $\bullet 2.82$, 36.28 .

München, Herbst 1899.

\section{Ferdinand Henrioh: Ueber die Conetitution des Mononitrosooroins.}

[Aus dem chemischen Institut der Universitat in Graz.]

(Eingegangen am 23. Norember.)

Bei Betrachtungen über die Constitution der zwei Modificationen des Mononitrosoorcins ${ }^{1}$ ) ging ich seinerzeit ron der Formel:

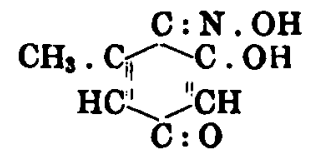

ans. Dieselbe war damals der allgemein übliche Ansdruck für die Constitution dieser Verbindung ${ }^{2}$ ). Das Mononitrosoorcin erscheint darnach als das Oxim eines Oxy-para-toluchinons s). - Nunmebr bat das Studium des Monomethylatbers des Nitrosoorcins ergeben, dass das Mononitrosoorcin sich ron einem Oxy-ortho-toluchinon ableitet.

Der Monomethyläther des Nitrosoorcins entsteht einerseits beim Kochen des Letzteren mit 3-procentiger methylalkoholischer Salzsăure (), andererseits durch Einwirkung von salpetriger Sāure auf den Monomethylăther des Orcins ${ }^{\jmath}$ ). Bei der zuletzt erwähnten Reaction

1) Monatshefte för Chemie etc. Wien, Bd. 18, 142 (1897).

7) S. Roscoe und Schorlemmer's ausführliches Lehrbuch der Chemie, Bd. 4, Theil 2, S. 378.

7) Ueber den Beweis, dass Mononitrosooroin ein Oxim, and kein wirklicher Nitrosolórper ist, siehe Monatshefte 18, 183.

ஒ) Monatshefte 18, 179 (1897). $\quad$ b) ibid. 18, 176. 